\title{
National Analysis of Network Drug Information Centers: Education, Training and Related Cost at Ministry of Health Hospitals in Saudi Arabia
}

\author{
Yousef Ahmed Alomi" ${ }^{*}$, Amani Abdullah Bahdailah², Arwa Kefah Alohaly ${ }^{3}$ \\ ${ }^{1}$ The Former General Manager of General Administration of Pharmaceutical Care and Former Head, National Clinical Pharmacy \\ and Pharmacy Practice and Pharmacy $R \&$ D Administration, Ministry of Health, Riyadh, SAUDI ARABIA. \\ ${ }^{2}$ Head, Pharmacy Education and Training Section, TPN \& Surgery Clinical Pharmacist, Pharmaceutical Care Department, King \\ Abdullah Bin Abdul-Aziz University Hospital Princess Noura University, Riyadh, SAUDI ARABIA. \\ ${ }^{3}$ Dental Doctor, Riyadh Elm University, Dental College, Riyadh, SAUDI ARABIA.
}

\section{Received: 02 October 2018; \\ Accepted: 26 November 2018 \\ *Correspondence to: \\ Dr. Yousef Ahmed Alomi,} The Former General Manager of General Administration of Pharmaceutical Care, Former Head, National Clinical Pharmacy and Pharmacy Practice Head, Pharmacy $R$ and $D$ Administration Ministry of Health, Riyadh,SAUDI ARABIA. Email:yalomi@gmail.com

Copyright: (c) the author(s),publisher and licensee Indian Academy of Pharmacists. This is an open-access article distributed under the terms of the Creative Commons Attribution Non-Commercial License, which permits unrestricted non-commercial use, distribution, and reproduction in any medium, provided the original work is properly cited.

\begin{abstract}
Objectives: To explore analysis of network Drug Information Education and training services with the related cost at Ministry of Health $(\mathrm{MOH})$ institutions. Methods: This is a simulation of a 2-months crosssectional survey of all drug information centers at $\mathrm{MOH}$ Hospitals. Any drug opened and provided services to health care professionals and the public participated in the questionnaire. All type of drug information centers national, regional or local at healthcare institutions including in the survey. All type of hospitals or primary care centers included in the survey (public, pediatric, maternity, psychiatry) included in the study. The survey consisted of two part. Demographics data and questions about education and training activity of drug information centers. It included education and training programs and activities for pharmacy staff and healthcare professional and training services for pharmacy student, pharmacy technician students and pharmacy residency programs with related cost analysis. All analysis done by using an electronic Survey Monkey system. Results: The survey distributed to sixty drug information centers with the total responded forty-six center, the response rate was $76.66 \%$. The majority of hospitals 11 (23.9\%) with (100-199) and 11 (23.9\%) with (200-299 beds). In the pharmacy training programs, the highest workload and cost implicated was Pharm. D or Master Pharmacist on job training programs (1.34 FTE) with average daily cost was (170.12 USD). The highest daily workload and cost of education and training activities delivered to the healthcare professional was short education courses $1-5$ days ( $0.72 \mathrm{FTE}$ ) with average daily cost was (91.53 USD). The highest daily workload and cost of education and training activities delivered by Drug Information Centers to Pharmacy staff was specialized Pharmacist Residency Program needed (6.56 FTE) with average daily cost was (832.13 USD). The highest workload and cost of education and training activities of drug information staff was specialized Pharmacist Residency Program needed (3.42 FTE) with average daily cost was (433.89 USD). Conclusion: One-third of the network drug information centers had education and training services despite the deficiency of the staff. Targeting of expanding the training services are highly recommended to cover the demand for hospital services.
\end{abstract}

Key words: Network, Drug Information centers, Education, Training, Cost, Ministry of Health, Saudi Arabia.

\section{INTRODUCTION}

The Pharmacy Education and Training is a potential element in the Pharmacy Practice. It required as minimum standards of hospital pharmacy, the ambulatory care services and drug information services. ${ }^{[1-3]}$ In addition to all Pharmaceutical services. The drug information centers provide several education and training sessions and programs. Those education and training services provided to the drug information centers staff, pharmacy staff and healthcare professional. The workload analysis and cost-related issues are required to fit with Saudi vision 2030..$^{[4-5]}$ Several published distrusted about drug information related cost avoidance. ${ }^{[6-12]}$ However, it challenging to find the workload analysis and workforce required investigation around the world. In addition to cost analysis of education and training activities of drug information centers. The objective is to explore the workload and cost analysis for education and training services of network drug information centers in Kingdom of Saudi Arabia (KSA).

\section{MATERIALS AND METHODS}

\section{Methods}

It is a simulation including a 2-months cross-sectional survey of all drug information centers at $\mathrm{MOH}$ hospitals. Any drug opened and provided services to health care professionals and the public participated in the questionnaire. All type of drug information centers national or regional or local at healthcare institutions including in the survey. All type of hospitals or primary care centers included in the survey (public, pediatric, maternity, psychiatry) included in the study. The survey consisted of two part. Demographics data and consisted of several part questions; Part one: workload of drug information centers, Part two: cost analysis of drug information centers foundations, Part three: the cost analysis of drug information activity and Part four: questions about education and training activity of drug information centers. The third one discussed in this study. It included education and training programs and activities for pharmacy 
staff and healthcare professional and training services for pharmacy student, pharmacy technician students and pharmacy residency programs with related cost analysis. All analysis was done by using an electronic Survey Monkey system.

\section{RESULTS}

The survey distributed to sixty drug information centers with the total responded forty-six center, the response rate was $76.66 \%$. The majority of hospitals 11 (23.9\%) with (100-199 beds) and 11 (23.9\%) with (200-299 beds). The majority of hospitals accredited of Saudi Center for Accreditation of Healthcare Institutions (CBAHI) 27 (58.7\%) and Saudi Commission of Health Specialties $9(19.6 \%)$ while $11(23.9 \%)$ hospitals not accredited by any organization. Most of the type of drug information centers were adults drug information centers $20(43.48 \%)$ and $15(32.61 \%)$ followed by psychiatric drug information centers $5(10.87 \%)$ and pediatrics drug information centers $4(8.7 \%)$ as explored in Table 1 . Among the responders the $43(95.6 \%)$ was Saudi and $2(4.4 \%)$ was non-Saudi. The age distribution (18-40 years) was high value $44(95.7 \%)$. The most educational level of the responders was Bachelor degree of Pharmacy 22 (47.83\%), Doctor of pharmacy 11 (23.9\%) and Master of Science degree 9 (19.57\%). Only $4(10 \%)$ has certified Pharmaceuticals Specialties. Most of responders $30(65.2 \%)$ were 1-6 years' experience with drug information services and $8(17.39 \%)$ of clinical pharmacy experiences as explored in Table 2 and Table 3. In the pharmacy training programs, the

\section{Table 1: Demographic hospital information.}

\begin{tabular}{|c|c|c|}
\hline Number of beds at the hospital & $\begin{array}{l}\text { Response } \\
\text { Count }\end{array}$ & $\begin{array}{l}\text { Response } \\
\text { Percent }\end{array}$ \\
\hline$<50$ & 7 & $15.2 \%$ \\
\hline $50-99$ & 6 & $13.0 \%$ \\
\hline $100-199$ & 11 & $23.9 \%$ \\
\hline $200-299$ & 11 & $23.9 \%$ \\
\hline $300-399$ & 4 & $8.7 \%$ \\
\hline $400-499$ & 4 & $8.7 \%$ \\
\hline$=$ or $>600$ & 3 & $6.5 \%$ \\
\hline Medical City & 0 & $0.0 \%$ \\
\hline Answered question & 46 & \\
\hline Skipped question & 0 & \\
\hline The hospital accreditation & Response Count & Response Percent \\
\hline CIBAHI & 27 & $58.7 \%$ \\
\hline Joint Commotion the USA & 7 & $15.2 \%$ \\
\hline Canada & 1 & $2.2 \%$ \\
\hline Saudi commission on health accreditation & 9 & $19.6 \%$ \\
\hline Non accredited & 11 & $23.9 \%$ \\
\hline Answered question & 46 & \\
\hline Skipped question & 0 & \\
\hline The type of drug information center & Response Count & Response Percent \\
\hline General Drug Information Center & 15 & $32.61 \%$ \\
\hline Adult drug information center & 20 & $43.48 \%$ \\
\hline Pediatric drug information center & 4 & $8.70 \%$ \\
\hline Psychiatric drug information center & 5 & $10.87 \%$ \\
\hline Oncology drug information center & 0 & $0.00 \%$ \\
\hline Cardiology drug information center & 1 & $2.17 \%$ \\
\hline Dental drug information center & 0 & $0.00 \%$ \\
\hline Primary health care drug information center & 1 & $2.17 \%$ \\
\hline Answered question & 46 & \\
\hline Skipped question & 0 & \\
\hline
\end{tabular}

highest workload and cost implicated was Pharm. D or Master pharmacist on job training programs (1.34 FTE) with average daily cost was (170.12 USD). Followed by Pharm. D or Master Student pharmacist-training program (1.18 FTE) with average daily cost was (149.32 USD) and Pharmacist Residency Program (1.07 FTE) with average daily cost (135.86 USD) as explored in Table 4. The highest daily workload and cost of education and training activities delivered to the healthcare professional was short education courses

\begin{tabular}{|c|c|c|}
\hline Nationality & $\begin{array}{l}\text { Response } \\
\text { Count }\end{array}$ & $\begin{array}{l}\text { Response } \\
\text { Percent }\end{array}$ \\
\hline Saudi & 43 & $95.6 \%$ \\
\hline Non-Saudi & 2 & $4.4 \%$ \\
\hline Answered question & 45 & \\
\hline Skipped question & 1 & \\
\hline Gender & $\begin{array}{l}\text { Response } \\
\text { Count }\end{array}$ & $\begin{array}{l}\text { Response } \\
\text { Percent }\end{array}$ \\
\hline $18-40$ years & 44 & $95.7 \%$ \\
\hline $40-65$ years & 2 & $4.3 \%$ \\
\hline $18-40$ years & 0 & $0.0 \%$ \\
\hline more than 65 years & 0 & $0.0 \%$ \\
\hline Answered question & 46 & \\
\hline Skipped question & 0 & \\
\hline Academic Qualification (s): & $\begin{array}{l}\text { Response } \\
\text { Count }\end{array}$ & $\begin{array}{l}\text { Response } \\
\text { Percent }\end{array}$ \\
\hline Diploma Pharmacy & 7 & $15.22 \%$ \\
\hline Bsc. Pharm & 22 & $47.83 \%$ \\
\hline M.S & 9 & $19.57 \%$ \\
\hline Msc. Clinical Pharmacy & 6 & $13.04 \%$ \\
\hline Pharm.D. & 11 & $23.91 \%$ \\
\hline Ph.D & 0 & $0.00 \%$ \\
\hline MBA & 3 & $6.52 \%$ \\
\hline Pharmacy Residency Two years (R1) & 1 & $2.17 \%$ \\
\hline Pharmacy Residency one year (R2) & 0 & $0.00 \%$ \\
\hline Fellowship & 1 & $2.17 \%$ \\
\hline Others & 1 & $2.17 \%$ \\
\hline Answered question & 46 & \\
\hline Skipped question & 0 & \\
\hline Total years worked as a pharmacist & $\begin{array}{l}\text { Response } \\
\text { Count }\end{array}$ & $\begin{array}{l}\text { Response } \\
\text { Percent }\end{array}$ \\
\hline $\begin{array}{l}\text { Board Certified Ambulatory Care Pharmacist } \\
\text { (BCACP) }\end{array}$ & 0 & $0.0 \%$ \\
\hline $\begin{array}{l}\text { Board Certified Critical Care Pharmacist } \\
\text { (BCCCP) }\end{array}$ & 0 & $0.0 \%$ \\
\hline Board Certified Nuclear Pharmacist (BCNP) & 1 & $2.5 \%$ \\
\hline $\begin{array}{l}\text { Board Certified Nutrition Support Pharmacist } \\
\text { (BCNSP) }\end{array}$ & 0 & $0.0 \%$ \\
\hline Board Certified Oncology Pharmacist (BCOP) & 0 & $0.0 \%$ \\
\hline $\begin{array}{l}\text { Board Certified Pediatric Pharmacy Specialist } \\
\text { (BCPPS) }\end{array}$ & 1 & $2.5 \%$ \\
\hline $\begin{array}{l}\text { Board Certified Pharmacotherapy Specialists } \\
\text { (BCPS) }\end{array}$ & 1 & $2.5 \%$ \\
\hline Board Certified Psychiatric Pharmacist (BCPP) & 1 & $2.5 \%$ \\
\hline Non & 39 & $97.5 \%$ \\
\hline Others & 1 & $2.5 \%$ \\
\hline Answered question & 40 & \\
\hline Skipped question & 6 & \\
\hline
\end{tabular}




\begin{tabular}{|l|l|l|l|l|l|}
\hline \multicolumn{2}{|l|}{ Table 3: Total years of experience. } \\
\hline $\begin{array}{l}\text { Answer } \\
\text { Options }\end{array}$ & $\begin{array}{l}\text { Pharmacy } \\
\text { Practice }\end{array}$ & $\begin{array}{l}\text { Clinical } \\
\text { Pharmacy }\end{array}$ & $\begin{array}{l}\text { Pharmacy } \\
\text { Administration }\end{array}$ & $\begin{array}{l}\text { Drug information } \\
\text { services }\end{array}$ & $\begin{array}{l}\text { Response } \\
\text { Count }\end{array}$ \\
\hline 0 & 2 & 7 & 3 & 3 & 13 \\
\hline$<1$ year & 3 & 5 & 4 & 6 & 16 \\
\hline $1-3$ & 8 & 5 & 9 & 14 & 24 \\
\hline $4-6$ & 17 & 3 & 7 & 16 & 28 \\
\hline$>6$ years & 23 & 5 & 11 & 8 & 29 \\
\hline answered question & & & 46 \\
\hline skipped question & & & & 0 \\
\hline
\end{tabular}

\section{Table 4: Workload and cost of Pharmacy training programs.}

\begin{tabular}{|c|c|c|c|c|c|c|c|c|c|c|c|c|c|}
\hline \multirow[b]{2}{*}{ Type of training programs } & \multicolumn{5}{|c|}{$\begin{array}{l}\text { The number of monthly trainees } \\
\text { per each program }\end{array}$} & \multicolumn{6}{|c|}{$\begin{array}{l}\text { The time spent for each trainee daily for } \\
\text { each program }\end{array}$} & \multirow[b]{2}{*}{ 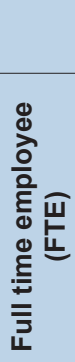 } & \multirow[b]{2}{*}{ 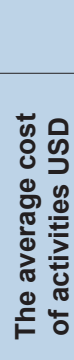 } \\
\hline & 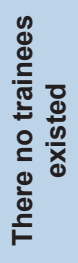 & 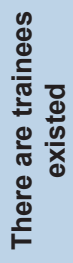 & 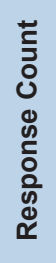 & 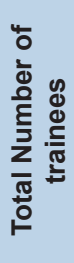 & 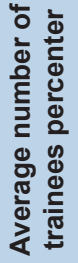 & 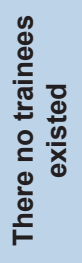 & 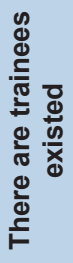 & 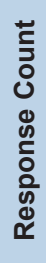 & 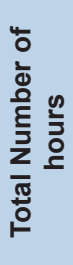 & 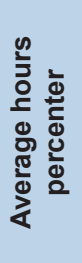 & 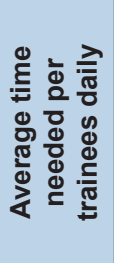 & & \\
\hline Pharmacy Technician students Training Program & 26 & 19 & 45 & 54 & 2.84 & 20 & 26 & 46 & 75.25 & 2.89 & 8.23 & 1.03 & 130.38 \\
\hline Pharmacy Technician on Job training Program & 19 & 23 & 42 & 67 & 2.91 & 20 & 24 & 44 & 58.75 & 2.45 & 7.13 & 0.89 & 113.02 \\
\hline Pharmacist Student Training Program & 22 & 22 & 44 & 66 & 3.00 & 20 & 24 & 44 & 62.25 & 2.59 & 7.78 & 0.97 & 123.33 \\
\hline Pharmacist on Job Training Program & 19 & 24 & 43 & 65 & 2.71 & 19 & 24 & 43 & 50.25 & 2.09 & 5.67 & 0.71 & 89.88 \\
\hline Pharmacist Residency Program & 40 & 3 & 43 & 16 & 5.33 & 37 & 7 & 44 & 11.25 & 1.61 & 8.57 & 1.07 & 135.86 \\
\hline $\begin{array}{l}\text { Pharm D OR Master Pharmacist Student Training } \\
\text { Program }\end{array}$ & 33 & 10 & 43 & 38 & 3.80 & 32 & 12 & 44 & 29.75 & 2.48 & 9.42 & 1.18 & 149.32 \\
\hline $\begin{array}{l}\text { Pharm D OR Master Pharmacist on Job Training } \\
\text { Program }\end{array}$ & 37 & 6 & 43 & 16 & 2.67 & 34 & 10 & 44 & 40.25 & 4.03 & 10.73 & 1.34 & 170.12 \\
\hline Pharm D OR Master Residency Program & 38 & 5 & 43 & 11 & 2.20 & 37 & 7 & 44 & 22.25 & 3.18 & 6.99 & 0.87 & 110.84 \\
\hline
\end{tabular}

1-5 days (0.72 FTE) with average daily cost was (91.53 USD). Followed by long reading session 4-5 weeks needed ( $0.61 \mathrm{FTE})$ with average daily cost was (76.83 USD) and weekly continuous medical education lecture needed (0.37 FTE) with average daily cost (47.32 USD) as explored in Table 5. The highest daily workload and cost of education and training activities delivered by Drug Information Centers to pharmacy staff was specialized pharmacist residency program needed (6.56 FTE) with average daily cost was (832.13 USD). Followed by long training session needed (1.05 FTE) with average daily cost was (133.71 USD) and general pharmacist residency program required (0.91 FTE) with average daily cost (115.57 USD) as explored in Table 6. The highest workload and cost of education and training activities of drug information staff was specialized pharmacist residency program needed (3.42 FTE) with average daily cost was (433.89 USD). Followed by conferences and symposium outside the Kingdom of Saudi Arabia needed (2.31 FTE) with average daily cost was (293.06 USD) and workshop outside the Kingdom of Saudi Arabia demanding (1.53 FTE) with average daily cost was (193.72 USD) as explored in Table 7.

\section{DISCUSSION}

The third pharmacy strategic goal at Ministry of Health organizations was related Pharmacy Human Resources and related issues. ${ }^{[13]}$ The general administration of Pharmaceutical Care conducted several educational sessions related to Pharmacy Practice and Clinical Pharmacy and the Drug Information Services were part of them. ${ }^{[13]}$ However, the workload of education and training activities and cost related not known. The author tried to explore the workload analysis of drug information centers and cost related to Ministry of Health in Kingdom of Saudi Arabia. The overall average of existing drug information education and training program was one-third of the subjects. That is results lower than what reported by Rosenberg, JM et al. That is related to the new network of drug information services at Ministry of Health. ${ }^{[14-15]}$ The results showed the highest workload education and training in drug information services was a pharmacist on job training and pharmacy student training program because it will more time and subsequently, the cost related. The highest workload and cost of education and training programs delivered to healthcare professional was short or long course and weekly lecture. Although short courses or weekly lecture is a short time, the more frequently 


\begin{tabular}{|c|c|c|c|c|c|c|c|c|c|c|c|c|c|}
\hline \multirow[b]{2}{*}{ Type of events } & \multicolumn{5}{|c|}{$\begin{array}{l}\text { The frequency of drug information } \\
\text { center delivers the Education and } \\
\text { Training to Healthcare professional } \\
\text { staff monthly }\end{array}$} & \multicolumn{8}{|c|}{$\begin{array}{l}\text { Time consumed by drug information center } \\
\text { to deliver the Education and Training to } \\
\text { Healthcare professional staff daily. }\end{array}$} \\
\hline & 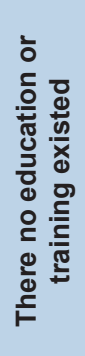 & 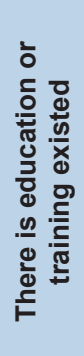 & 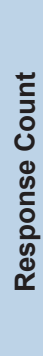 & 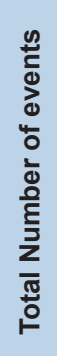 & 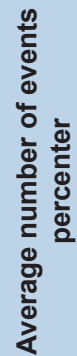 & 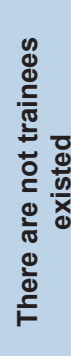 & 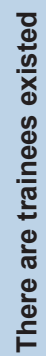 & 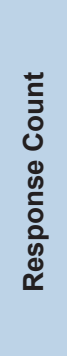 & 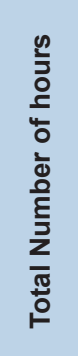 & 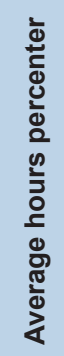 & 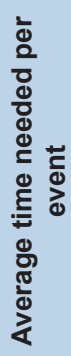 & 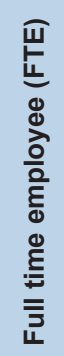 & 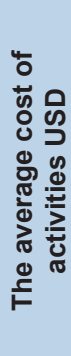 \\
\hline Weekly lecture CME & 22 & 22 & 44 & 68 & 3.09 & 22 & 22 & 44 & 21.25 & 0.97 & 2.99 & 0.37 & 47.32 \\
\hline Short education course $1-5$ days & 22 & 20 & 42 & 42 & 2.10 & 21 & 19 & 40 & 52.25 & 2.75 & 5.78 & 0.72 & 91.53 \\
\hline Long training session 4-5 weeks & 31 & 11 & 42 & 23 & 2.09 & 30 & 11 & 41 & 25.5 & 2.32 & 4.85 & 0.61 & 76.83 \\
\hline Orientation program & 18 & 24 & 42 & 37 & 1.54 & 24 & 18 & 42 & 21.25 & 1.18 & 1.82 & 0.23 & 28.85 \\
\hline Basic Medication Safety & 15 & 26 & 41 & 64 & 2.46 & 18 & 24 & 42 & 26.5 & 1.10 & 2.72 & 0.34 & 43.08 \\
\hline Cardiopulmonary Resusitation Medications & 24 & 17 & 41 & 31 & 1.82 & 23 & 18 & 41 & 29 & 1.61 & 2.94 & 0.37 & 46.57 \\
\hline Intravenous Medications & 25 & 17 & 42 & 44 & 2.59 & 24 & 17 & 41 & 15.75 & 0.93 & 2.40 & 0.30 & 38.01 \\
\hline Emergency Medications & 21 & 21 & 42 & 43 & 2.05 & 22 & 19 & 41 & 25.25 & 1.33 & 2.72 & 0.34 & 43.13 \\
\hline Distance learning CME education by pharmacy & 31 & 10 & 41 & 21 & 2.10 & 26 & 14 & 40 & 16.75 & 1.20 & 2.51 & 0.31 & 39.82 \\
\hline
\end{tabular}

\begin{tabular}{|l|l|l|l|l|l|l|l|l|l|l|l|l|l|}
\hline \multicolumn{7}{|c|}{ Table 6: Workload and cost of drug information center deliver the Education and Training to pharmacy staff. } \\
\hline \\
\hline
\end{tabular}




\begin{tabular}{|c|c|c|c|c|c|c|c|c|c|c|c|c|c|}
\hline \multirow[b]{2}{*}{ Type of events } & \multicolumn{5}{|c|}{$\begin{array}{l}\text { The frequency of drug information } \\
\text { center staff Share at Education } \\
\text { and Training monthly }\end{array}$} & \multicolumn{6}{|c|}{$\begin{array}{l}\text { drug information staff share consumes the } \\
\text { time at Education and Training staff daily }\end{array}$} & \multirow[b]{2}{*}{ 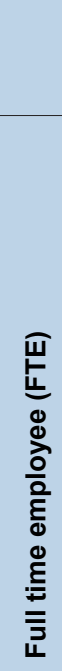 } & \multirow[b]{2}{*}{ 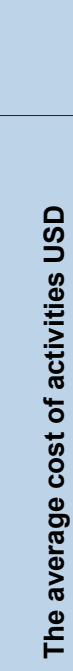 } \\
\hline & 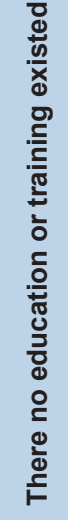 & 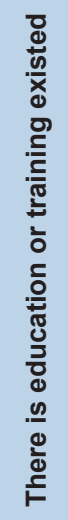 & 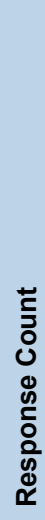 & 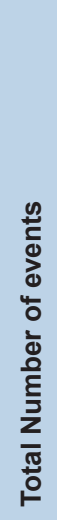 & 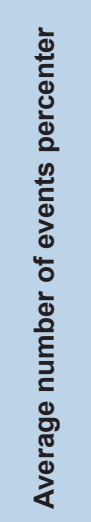 & 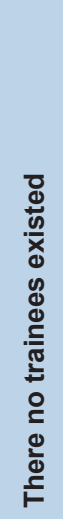 & 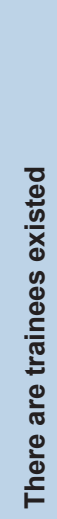 & 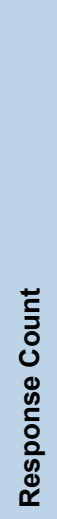 & 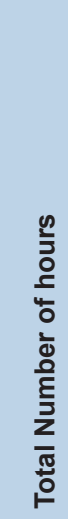 & 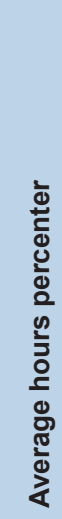 & 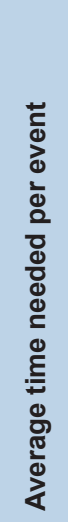 & & \\
\hline Weekly lecture CME & 17 & 25 & 42 & 58 & 2.32 & 19 & 23 & 42 & 27.25 & 1.18 & 2.75 & 0.34 & 43.57 \\
\hline Short education course $1-5$ days & 20 & 23 & 43 & 48 & 2.09 & 17 & 23 & 40 & 57.25 & 2.49 & 5.19 & 0.65 & 82.34 \\
\hline $\begin{array}{l}\text { Long training session } \\
4-5 \text { weeks }\end{array}$ & 24 & 17 & 41 & 38 & 2.24 & 23 & 15 & 38 & 40.25 & 2.68 & 6.00 & 0.75 & 95.07 \\
\hline General Pharmacist residency program & 38 & 3 & 41 & 21 & 7.00 & 35 & 4 & 39 & 10.25 & 2.56 & 17.94 & 2.24 & 284.31 \\
\hline Specialized pharmacist residency program & 38 & 4 & 42 & 18 & 4.50 & 36 & 3 & 39 & 18.25 & 6.08 & 27.38 & 3.42 & 433.89 \\
\hline Distance learning pharmacy education & 27 & 15 & 42 & 35 & 2.33 & 29 & 10 & 39 & 17.25 & 1.73 & 4.03 & 0.50 & 63.80 \\
\hline Conference and Symposium inside KSA & 15 & 25 & 40 & 65 & 2.60 & 17 & 22 & 39 & 83.25 & 3.78 & 9.84 & 1.23 & 155.94 \\
\hline Conference and Symposium outside KSA & 31 & 10 & 41 & 31 & 3.10 & 32 & 7 & 39 & 41.75 & 5.96 & 18.49 & 2.31 & 293.06 \\
\hline Workshop inside KSA & 15 & 25 & 40 & 66 & 2.64 & 18 & 21 & 39 & 87.5 & 4.17 & 11.00 & 1.38 & 174.35 \\
\hline Workshop outside KSA & 33 & 9 & 42 & 30 & 3.33 & 33 & 6 & 39 & 22 & 3.67 & 12.22 & 1.53 & 193.72 \\
\hline
\end{tabular}

than the long course. All them the drug information pharmacist spent more time and cost by them. While the workload of education and training activities delivered to pharmacy staff was residency program or long-term session and this was normal because more of pharmacist got training rather than courses or lecture, so the drug information pharmacist spent more time and cost related with those type of training and education. The highest workload of training or education delivered to pharmacy staff was specialized residency or education as conferences inside or outside Saudi Arabia. That is finding expected because most of drug information pharmacist need specialized residency or participation in the conferences. That has were not received shout courses or long course much or weekly lectures. All previous results were hard to compare them because of insufficiently published literature. The study explored all workload and related cost training or education services delivered by drug information services. It very useful during utilized them by other hospitals or organizations and fit by Saudi Vision 2030 or Ministry of Heath with privatization. ${ }^{[4-5]}$

\section{CONCLUSION}

The training and education services of network drug information centers were reasonably cost. Targeting to expand the services will increase pharmacy revenue and fit with Saudi Vision 2030 and new healthcare at Ministry of Health organizations during the privatization.

\section{ACKNOWLEDGEMENT}

None.

\section{CONFLICT OF INTEREST}

None.

\section{ABBREVIATIONS}

KSA: Kingdom of Saudi Arabia; MOH: Ministry of Health.

\section{REFERENCES}

1. Brenner TS, Godwin HN, Gouveia WA, Hodgkins BD, Kent SS, Kienle PC, et al. ASHP guidelines: Minimum standard for pharmacies in hospitals. Am J Heal Pharm. 2013;70(18):1619-30.

2. Buxton JA, Babbitt RM, Clegg CA, Durley SF, Epplen KT, Marsden LM, et al. ASHP guidelines: Minimum standard for ambulatory care pharmacy practice. Am J Heal Pharm. 2015;72(14):1221-36.

3. Ghaibi S, Ipema H GM. ASHP Guideline on The Pharmacist's Role in Providing Drug Information. Am J Heal Pharm. 2015;72(7):573-7.

4. Government of Saudi Arabia. Saudi Arabia Vision 2030. 2016. Available from: file://C:/ Users/yalomi/Downloads/Saudi_Vision2030_EN (1).pdf

5. Council for Economic Affairs and Development in KSA. National Transformation Program 2020. 2016;113. Available from: http://vision2030.gov.sa/sites/default/files/ NTP_ar.pdf

6. Alomi YA, Almudaiheem HY, Alarnous T, Alshurei S, Alsharafa A, Alzahrani T, et al. Cost-Efficiency of National Drug Information Center Through Ministry of Health Hotline Calling Services (937) in Saudi Arabia: Application of a Mercian Model. Value Heal. 2015;18(7):A735.

7. Skoutakis VA, Wojciechowski NJ, Carter CA, Hayes JM, Hudson BL, Martin JA. Drug Information Center network: need, effectiveness and cost justification. Drug Intell Clin Pharm.1987;21(1):49-56. 
8. Lyrvall H, Nordin C, Jonsson E, Alvan G, Öhman B. Potential savings of consulting a drug information center. Annals of Pharmacotherapy. 1993;27(12):1540.

9. Kinky DE, Erush SC, Laskin MS, Gibson GA. Economic impact of a drug information service. Ann Pharmacother. 1999;33(1):11-6.

10. Marrone CM, Heck AM. Impact of a Drug Information Service: Practitioner Hours Saved. Hosp Pharm. 2000;35(10):1065-70.

11. Ponampalam R, Loh CS. Cost benefits of the Drug and Poison Information Centre in preventing unnecessary hospitalisation: The Singapore experience. Hong Kong $\mathrm{J}$ Emerg Med. 2010;17(1):45-53.
12. Brown JN. Cost Savings Associated With a Dedicated Drug Information Service in an Academic Medical Center. Hosp Pharm. 2011;46(9):680-4.

13. Alomi YA, Alghamdi SJ, Alattyh RA. Strategic Plan of General Administration of Pharmaceutical Care at Ministry of Health in Saudi Arabia 2012 - 2022. JPharm Pharm Sci. 2015;1(13):1-8.

14. Rosenberg JM, Koumis T, Nathan JP, Cicero LA, Mcguire H. Current status of pharmacist-operated drug information centers in the United States. Am J Heal Pharm. 2004;61(19):2023-32.

15. Rosenberg JM, Schilit S, Nathan JP, Zerilli T, Mcguire H. Update on the status of 89 drug information centers in the United States. Am J Heal Pharm. 2009;66(19):1718-22.

Cite this article as: Alomi YA, Bahdelah A, Alohaly AK. National Analysis of Network Drug Information Centers Education, Training with the Related Cost at MOH Hospitals in Saudi Arabia. J Pharm Pract Community Med. 2018;4(4):231-6. 